\section{Comparing genomic profiles of tumour and peritumoral biopsies to find new possible biomarkers for glioblastoma therapy}

Martina Giambra, ${ }^{1}$ Eleonora Messuti, ${ }^{1}$ Serena Redaelli, ${ }^{1}$ Andrea Di Cristofori, ${ }^{2}$ Angela Bentivegna 1

${ }^{1}$ Milan-Bicocca University, Monza e Brianza; 2San Gerardo Hospital, Monza e Brianza, Italy

\begin{abstract}
The study of glioblastoma genomic profiles permits the use of CNAs load to identify patients with poor prognosis and the understanding of the genomic signatures essential for the disease maintenance and the identification of new potential biomarkers.
\end{abstract}

\section{Introduction}

Glioblastoma Multiforme (GBM, G-IV) represents the most common and malignant brain tumour. ${ }^{1}$ Despite the aggressive current standard treatments, therapies are not curative and patients survive on average 1215 months from diagnosis, with $90 \%$ of cases relapse and a final mortality rate close to $100 \% .2,3$ Treatment failure is due to the extreme heterogeneity of GBM, which primarily reflects the coexistence in the Tumour Core (TC) of different cell populations, including the Glioma Stem Cells (GSCs), and secondly the contribution, still to be defined, of the peritumoral brain zone (PBZ). ${ }^{4}$

In this work we analyse the genomic profiles of tumour and peri-tumour biopsies of GBM patients, and of their relative cell cultures (short-term primary cultures), using comparative genomic hybridization array (array-CGH). These kinds of cultures are the most straightforward, reliable and reproducible in vitro model currently available as they derive from patients and resemble mostly with tumour-of-origin. In addition, the quantity of fictitious mutations is limited by few-passages of cell culture. Our aim is to identify the copy number alterations (CNAs) essential for tumour survival and usable as biomarkers of tumour progression and recurrence.

\section{Materials and Methods}

Primary cell cultures were isolated directly from the patients' biopsies.

GSCs cultures stemness characterization: clonal assay to demonstrate GSCs selfrenewal efficiency; IF with neural differentiation markers ( $\beta$ III Tubulin, GFAP and MBP) to evaluate GSCs multipotency capability.

Genomic analysis was performed by Array-CGH technology (SurePrint G3 Human CGH 8x60 K, Agilent Technologies, Santa Clara, CA, USA).

The Database for Annotation, Visualization and Integrated Discovery [(DAVID), v 6.8 https://david.ncifcrf.gov/summary.jsp] was used to analyse shared CNAs found in the samples.

\section{Results}

The preliminary analysis on $15 \mathrm{TCs}$ confirmed the intertumoral heterogeneity and a high percentage of copy number alterations (CNAs) in GBM canonical pathways. In particular, PIK3KC pathway was affected in $73 \%$, Rb pathway in $67 \%$ and p53 pathway in $20 \%$ of TCs. Twelve TCs have at least one imbalance in the GBM canonical altered pathways, while 3 TCs have none. In each patient, individual alterations were also observed. In addition, the CNA load correlates with a worst
Correspondence: Martina Giambra, MilanBicocca University, Monza e Brianza, Italy. E-mail:m.giambra1@campus.unimib.it

Key words: GBM; GSC; PBZ; CAN; arrayCGH.

Acknowledgments: The authors thank Clarissa Cavandoli for her support, and all the patients.

Disclosures: The authors have nothing to disclose.

Conference presentation: This paper was presented at the Third Centro 3R Annual Meeting - L'era delle 3R: modelli in silico, in vitro e in vivo per promuovere la ricerca traslazionale 30 September - 1 October 2021, Evento online organizzato dal Politecnico di Torino.

Received for publication: 9 July 2021.

Accepted for publication: 7 September 2021.

This work is licensed under a Creative Commons Attribution NonCommercial 4.0 License (CC BY-NC 4.0).

CCopyright: the Author(s), 2021

Licensee PAGEPress, Italy

Biomedical Science and Engineering 2021; 4(s1):170 doi:10.4081/bse.2021.170

\section{prognosis (Table.1).}

We obtained 9 GSC cultures from TCs biopsies and 4 PBZ cultures from peritumoral biopsies. Comparing the

Table 1. Presence of imbalances (+) found in GBM canonically altered pathways and CNA load in tumour core (TC) biopsies of each patient. Blue squares indicate patients without follow-up data; orange ones indicate patients with relapse; green ones indicate patients died of disease.

\begin{tabular}{c|c|c|c|c|}
$\mathbf{T C}$ & $\begin{array}{c}\mathbf{p 5 3} \\
\text { pathway }\end{array}$ & $\begin{array}{c}\mathbf{R B} \\
\text { pathway }\end{array}$ & $\begin{array}{c}\text { PIK3KC } \\
\text { pathway }\end{array}$ & $\begin{array}{c}\text { Total } \\
\mathbf{C N A s}\end{array}$ \\
\hline 1 & & & & 1 \\
\hline 2 & & + & & 50 \\
\hline 3 & & & + & 5 \\
\hline 4 & & + & + & 52 \\
\hline 6 & & & & 11 \\
\hline 7 & & + & + & 27 \\
\hline 8 & & + & + & 19 \\
\hline 9 & & + & + & 16 \\
\hline 10 & + & + & + & 55 \\
\hline 11 & & + & + & 25 \\
\hline 12 & & + & + & 27 \\
\hline 13 & & + & + & 14 \\
\hline 15 & & & & 18 \\
\hline 17 & + & + & + & 24 \\
\hline 20 & + & + & + & 19 \\
\hline
\end{tabular}





Figure 1. Examples of CNAs distribution by chromosome a for TC (red plot) and matched GSC (blue plot) of patient number 7; b for TC (red plot) and matched PBZ (green plot) of patient number 11 .

genomic profiles of 9 TCs with the matched GSCs we evidenced a robust similarity, albeit with an evident clonal selection (Figure 1a). Furthermore, a correlation is observed between the percentage of shared CNAs and worst prognosis.

The comparison between the genomic profiles of 7 TCs with their matched PBZs did not show a good sharing of CNAs, except for 3 cases, highlighting PBZ exclusive alterations and a possible correlation between PBZ CNA load and worst prognosis (Figure 1b).

Similarly, the genomic profiles of 4 PBZs and the relative PBZ cells, did not show a good correlation. The same emerged from the comparison between the genomic profiles of the two types of cell cultures, GSCs and PBZ cells.

Using DAVID, we identified an impairment of pathways related to the development and progression of cancer and focal adhesion in the CNAs shared among our samples.

\section{Discussion and Conclusions}

In conclusion, this work allowed us to perform a holistic study on the genomic profiles of tumour and peritumour biopsies of GBM and related primary cultures. The absence of a marked overlap of the genomic profiles between TC and the matched PBZ could indicate the presence of CNAs specific to the tumoral core and marginal space. Furthermore, although in a few analysed cases, an in vitro selection seems to be more stringent in the PBZ counterpart than in the tumoral one, since we have observed a minor overlap between PBZ biopsies and the related primary cultures. The data obtained represent a good starting point for future investigations. In fact, the load of genomic imbalances seems to correlate with a poorer prognosis and the molecular function of shared CNAs may be used to rank patients according to follow-up trends and develop specific target therapies.

\section{References}

1. Louis DN, Perry A, Reifenberger G, et al. The 2016 World Health Organization classification of tumors of the central nervous system: a summary. Acta Neuropathol 2016;131:803-20. 2.

Ricard D, Idbaih A, Ducray F, et al. Primary brain tumours in adults. Lancet 2012;379:1984-96.

3. Persano L, Rampazzo E, Della Puppa A, et al. The three-layer concentric model of glioblastoma: cancer stem cells, microenvironmental regulation, and therapeutic implications. Sci World J 2011;11:1829-41.

4. Lemée JM, Clavreul A, Menei P. Intratumoral heterogeneity in glioblastoma: don't forget the peritumoral brain zone. Neuro Oncol 2015;17:1322-32. 